\title{
Avaliação do risco cardiovascular - metodologias e suas implicações na prática clínica
}

Filipa Mafra,* Helena Oliveira*

\section{RESUMO}

Introdução: O risco de vir a sofrer de doença aterosclerótica e suas complicações cardiovasculares, designado por risco cardiovascular global, pode ser avaliado utilizando várias metodologias, baseadas em escalas de risco. Estas entram em linha de conta com diversos factores de risco, interactivos, e apresentam algumas vantagens e limitações que importa considerar. $O$ objectivo da avaliação do risco cardiovascular é identificar grupos de indivíduos que devem ser aconselhados e tratados com o intuito de prevenir a doença cardiovascular, bem como estabelecer o nível de agressividade da terapêutica a instituir. Incluem-se considerações acerca de grupos de risco com características particulares, nomeadamente, mulheres, idosos e diabéticos.

Metodologia: Foi realizada uma pesquisa nas bases de dados da Internet, consultadas fontes documentais de referência nas áreas da Cardiologia, Medicina Geral e Familiar e Medicina Interna, incluindo as recomendações de várias organizações internacionais relativas à avaliação do risco cardiovascular.

Conclusões: A importância crescente da mortalidade e da morbilidade por doença cardiovascular aterosclerótica torna a avaliação do risco cardiovascular global essencial na prática clínica actual. O cálculo deste risco deve ser regular e dinâmico, exigindo revisão sempre que haja alteração nos factores de risco presentes. O valor obtido deve ser alvo de ponderação pelo médico, considerando aspectos que não são tidos em conta nas escalas, utilizando o senso clínico e adequando as decisões ao indivíduo em causa. As escalas de risco existentes, apesar das limitações que têm, são instrumentos valiosos, contribuindo para decisões médicas assentes em estudos e baseadas na evidência.

Palavras-chave: Risco Cardiovascular Global; SCORE; Framingham; Risco Cardiovascular em Mulheres; Risco Cardiovascular em Diabéticos; Risco Cardiovascular em Idosos.

\section{INTRODUÇÃO}

A doença aterosclerótica tem uma natureza multifactorial. Na sua génese estão implicados factores de diversa ordem: genéticos, ambientais, hemodinâmicos, dietéticos, metabólicos, inflamatórios, etc. Tem uma evolução longa e progressiva.

A possibilidade de complicações cardiovasculares da doença aterosclerótica não depende apenas de um factor em particular, mas sim da presença concomitante de diversos factores de risco. O risco cardiovascular global é o risco obtido pela presença e interacção de todos os

*Assistentes de Medicina Geral e Familiar, Serviço de Médicos Assistentes do Centro Clínico Ambulatório dos SAMS-SBSI (Serviços de Assistência Médico-Social do Sindicato dos Bancários do Sul e llhas). factores de risco num indivíduo. A presença simultânea de vários factores de risco tem um efeito sinérgico e multiplicativo. Eles interagem e potenciam-se, pelo que o risco cardiovascular global é muito mais do que a soma do risco dado por cada um dos factores isolados.

O objectivo do cálculo do risco cardiovascular global é identificar os indivíduos que devem ser aconselhados e que devem receber tratamento para prevenir a doença cardiovascular, bem como estabelecer o nível de agressividade da terapêutica.

\section{A QUEM SE DEVE CALCULAR O RISCO CARDIOVASCULAR GLOBAL?}

O risco cardiovascular global deve ser calculado nos in- 
divíduos considerados em risco de desenvolver doença aterosclerótica, ou seja, em prevenção primária da doença cardiovascular. Estes indivíduos são os que têm factores de risco mas ainda sem doença estabelecida.

Os indivíduos com doença aterosclerótica estabelecida, são considerados de alto risco e devem ser abordados de forma intensiva, tratando todos os factores de risco agressivamente. Os doentes com Diabetes Mellitus (DM) tipo 2, os com Diabetes Mellitus tipo 1 com microalbuminúria, os indivíduos com níveis muito elevados de um único factor de risco, os com aneurisma da aorta abdominal ou com Insuficiência Renal Crónica são considerados, em termos de risco cardiovascular global, equivalentes aos doentes com doença cardiovascular estabelecida e devem ser abordados como de alto risco. ${ }^{1}$

Nos casos considerados de alto risco, o cálculo do risco cardiovascular global não é utilizado para avaliar o grau de risco, uma vez que esse já é conhecido, mas pode ser útil para avaliar o benefício das intervenções terapêuticas usadas, ao permitir comparar um valor prévio de risco com um segundo, obtido após terapêutica.

\section{COMO SE CALCULA O RISCO CARDIOVASCULAR?}

Para calcular o risco cardiovascular global, e de acordo com as European Guidelines on Cardiovascular Disease Prevention, ${ }^{1}$ deve fazer-se a avaliação dos seguintes parâmetros:

- Anamnese: história de doença cardiovascular prévia, história familiar de doença cardiovascular prematura, tabagismo, hábitos dietéticos, actividade física, nível social e educacional;

- Exame objectivo: pressão arterial, frequência cardíaca, auscultação cardio-pulmonar, palpação de pulsos periféricos, índice de massa corporal, perímetro da cintura, fundoscopia [se houver hipertensão arterial (HTA) grave];

- Exames laboratoriais: glicosúria, proteinúria, micro-albuminúria, colesterol total, lipoproteinas de elevada densidade (HDL), lipoproteinas de baixa densidade (LDL), triglicéridos, glicemia, creatininemia;

- Electrocardiograma, prova de esforço (se suspeita de angina);

- Ecocardiograma (se HTA);

- Proteína C reactiva (PCR) de alta sensibilidade, lipo- proteína a, fibrinogenemia, homocisteinemia (se doença cardiovascular prematura, severa ou história familiar de doença cardiovascular prematura).

Foram criadas escalas de avaliação do risco cardiovascular global, instrumentos que permitem quantificar o risco de um determinado indivíduo, resultante da presença simultânea de vários factores de risco cardiovascular.

O uso destas escalas vai permitir:

- Quantificar o risco e identificar quais os indivíduos com risco elevado;

- Avaliar a necessidade e a intensidade da intervenção terapêutica, ao estabelecer valores de risco a partir dos quais deve ser ponderada a introdução de terapêutica farmacológica, para além das medidas terapêuticas não farmacológicas;

- Motivar os indivíduos para alterar comportamentos, uma vez que são instrumentos muito úteis para explicar o conceito de risco cardiovascular, bem como as implicações de ter um risco elevado e ilustrar quais os benefícios passíveis de serem obtidos com a redução dos factores de risco existentes. A tomada de consciência e a co-responsabilização do doente pode assim ajudar a conseguir uma melhor aderência às medidas terapêuticas propostas;

- De acordo com o valor de risco obtido, definir intervalos de vigilância médica e estabelecer objectivos terapêuticos.

Existem várias escalas para cálculo do risco cardiovascular global. Abordaremos as escalas de Framingham (ver Figura 1 e 2) e SCORE (Systematic COronary Risk Evaluation) (ver Figura 3), pelas seguintes razões:

- A escala de Framingham, americana, teve uma importância muito grande no desenvolvimento do conceito de risco global e é a base da criação de muitas outras escalas, incluindo a usada no Adult Treatment Panel III (ATP III); ${ }^{2}$

- O SCORE é o instrumento recomendado na Europa e no nosso país, de acordo com a circular normativa da Direcção-Geral de Saúde, publicada em Abril de $2007 .^{3}$

\section{ESCALA DE FRAMINGHAM}

A escala de risco de Framingham foi construída com base no estudo Framingham, um estudo observacional 


\section{Coronary Disease Risk Prediction Score Sheet for Men Based on Total Cholesterol Level}
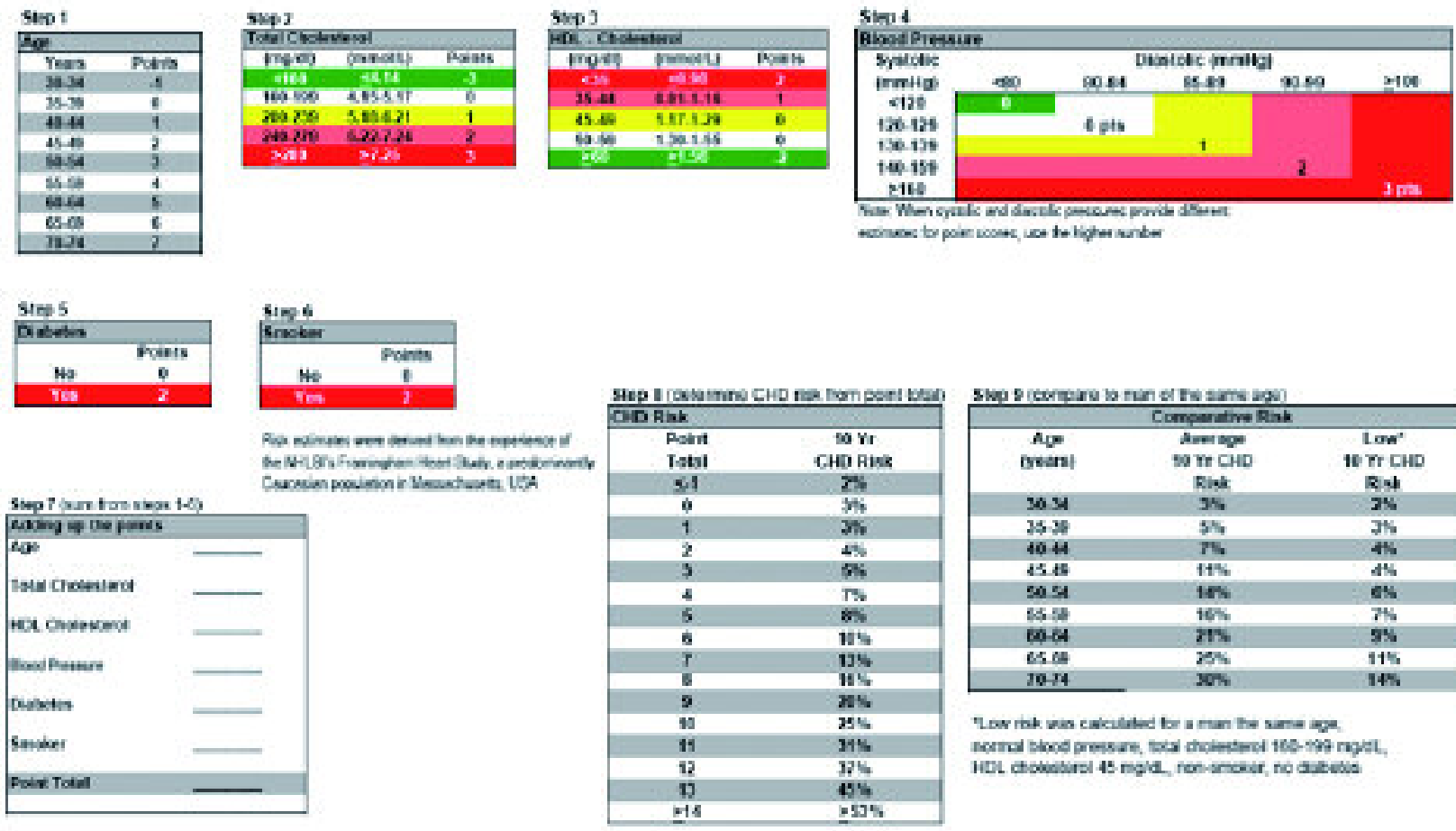

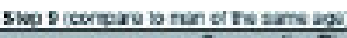

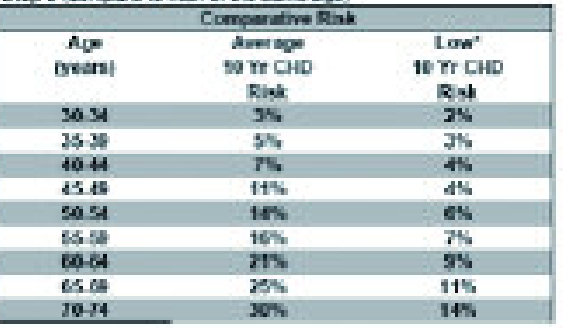

"Low ribk win calculated bre a man the same woe.

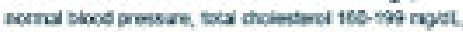

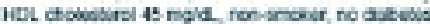

Figura 1. Tabela de cálculo de risco de Framigham para o sexo masculino, baseada no colesterol total.

prospectivo, que decorreu na pequena cidade do nordeste dos Estados Unidos da América (EUA), com o mesmo nome. ${ }^{3,4}$ Este estudo avaliou, durante 50 anos, a pressão arterial, tabagismo, perfil lipídico, e outras características, de 5.300 indivíduos, de diferentes etnias, residentes em Framingham, com idades entre os $30 \mathrm{e}$ os 74 anos à data da observação inicial, bem como as suas causas de morte e doença. Estes dados foram usados para calcular o risco absoluto de eventos coronários, fatais e não fatais, aos 10 anos. Esta escala avalia mortalidade e morbilidade por doença coronária [risco de angina, Enfarte Agudo do Miocárdio (EAM) e morte por doença coronária].

Foram criadas quatro folhas de cálculo do risco cardiovascular: uma com base no colesterol total e outra no colesterol LDL, para cada um dos sexos. Os parâmetros usados para o cálculo do risco cardiovascular global são, em categorias, a idade, o colesterol total, o colesterol HDL, a pressão arterial e ainda a presença ou não de diabetes e tabagismo. Um risco aos 10 anos su- perior ou igual a $20 \%$ foi arbitrariamente recomendado como o limite a partir do qual deverá haver uma intensa intervenção para modificação dos factores de risco. Um risco absoluto global aos 10 anos de $20 \%$ significa que, nos próximos 10 anos, em cem indivíduos com os mesmos factores de risco, vinte vão sofrer um evento coronário (fatal ou não fatal).

O limite de risco de $20 \%$ no Framingham tem sido muito criticado porque conduz a uma muito elevada prevalência de indivíduos com risco elevado nas faixas etárias mais altas, particularmente nos homens, e pode dar uma impressão errada do risco a longo prazo dos indivíduos jovens com factores de risco elevados. Recentemente, o estudo de Framingham já adoptou o conceito de doença coronária grave (inclui apenas EAM fatal e não fatal) o que já diminui o grau de risco em comparação com a definição anterior. É esta definição mais recente que é usada na escala de cálculo de risco cardiovascular global incluída no ATP III. ${ }^{5}$

Existe ainda uma tabela que permite o cálculo do 


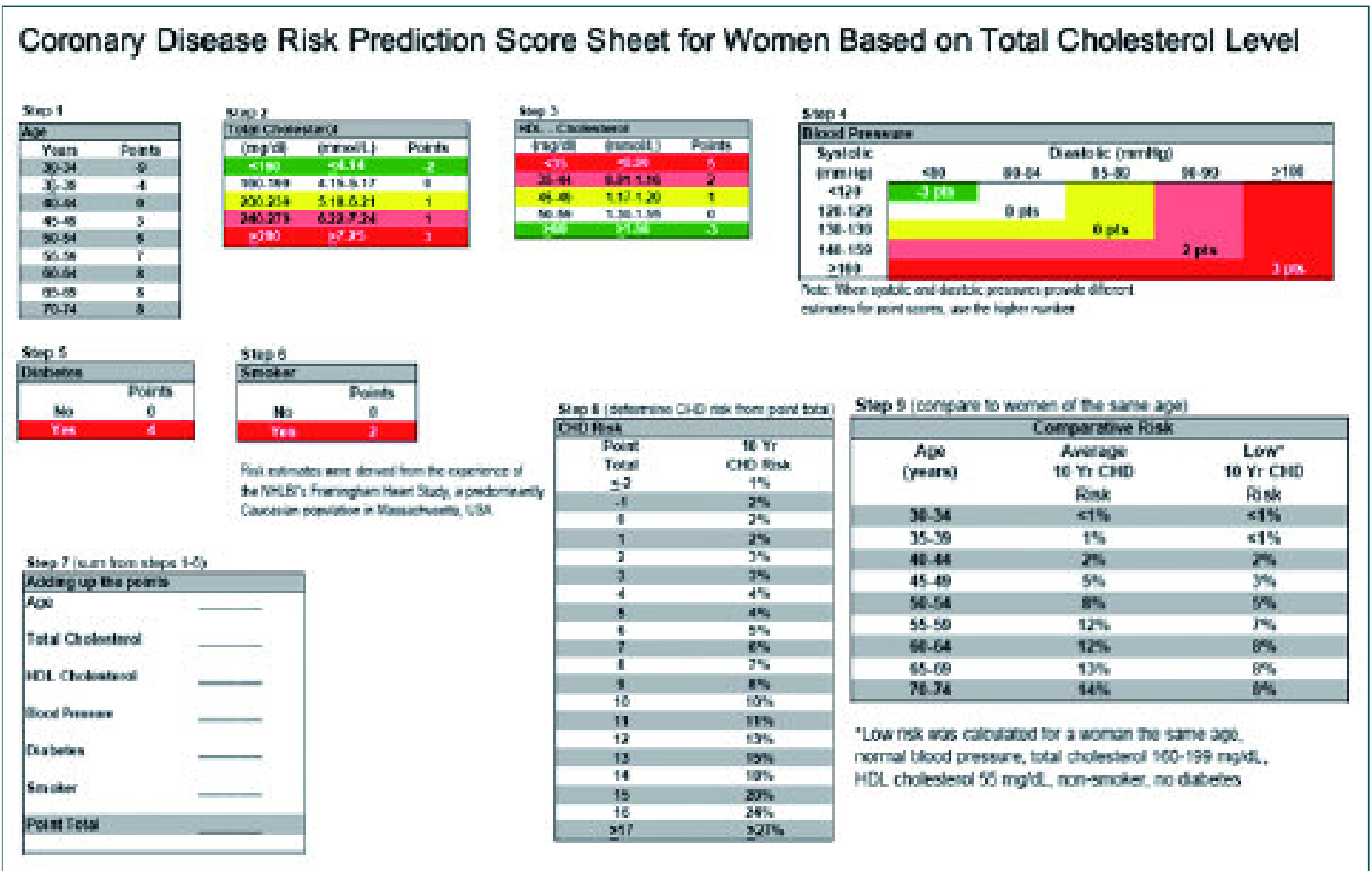

Figura 2. Tabela de cálculo de risco de Framigham para o sexo feminino, baseada no colesterol total.

risco relativo, ou seja, relaciona o valor obtido com o valor médio de risco para uma pessoa da mesma idade. O cálculo do risco relativo é importante porque a idade, só por si, constitui um factor de risco cardiovascular. Este facto assume particular relevância nos extremos etários, porque o risco absoluto tende a ser baixo nos jovens e elevado nos idosos. A riscos absolutos baixos pode corresponder riscos relativos elevados e vice-versa. Nos jovens, para além do cálculo do risco relativo, deve também extrapolar-se o risco para a idade de 60 anos, como forma de contornar o risco absoluto baixo que as idades jovens quase todas têm.

\section{Limitações da Escala de Framingham}

Considerando o estudo que serviu de base à construção desta escala, é possível identificar algumas limitações importantes: ${ }^{5}$

1. Reflecte a realidade de uma pequena população do nordeste americano. Sobrestima o risco de eventos cardiovasculares nas populações de baixo risco do sul da Europa, que apresentam características e estilos de vida muito diferentes. Para além do risco na Europa ser diferente do da população americana, dentro da própria Europa há grandes diferenças entre os países, em relação ao risco de EAM e Acidente Vascular Cerebral (AVC).

2. No estudo de Framigham havia poucos eventos para alguns factores de risco, pelo que pode haver falta de acuidade no risco calculado.

3. De igual forma, havia poucos eventos nas idades extremas, pelo que pode haver pouca acuidade no risco calculado para estas faixas etárias.

4. Havia poucas mulheres e sobretudo poucas mulheres idosas.

5. A maioria dos indivíduos era caucasiana, pelo que há dificuldade em extrapolar para outras raças ou etnias.

6. Não é possível combinar mais de cinco variáveis. 


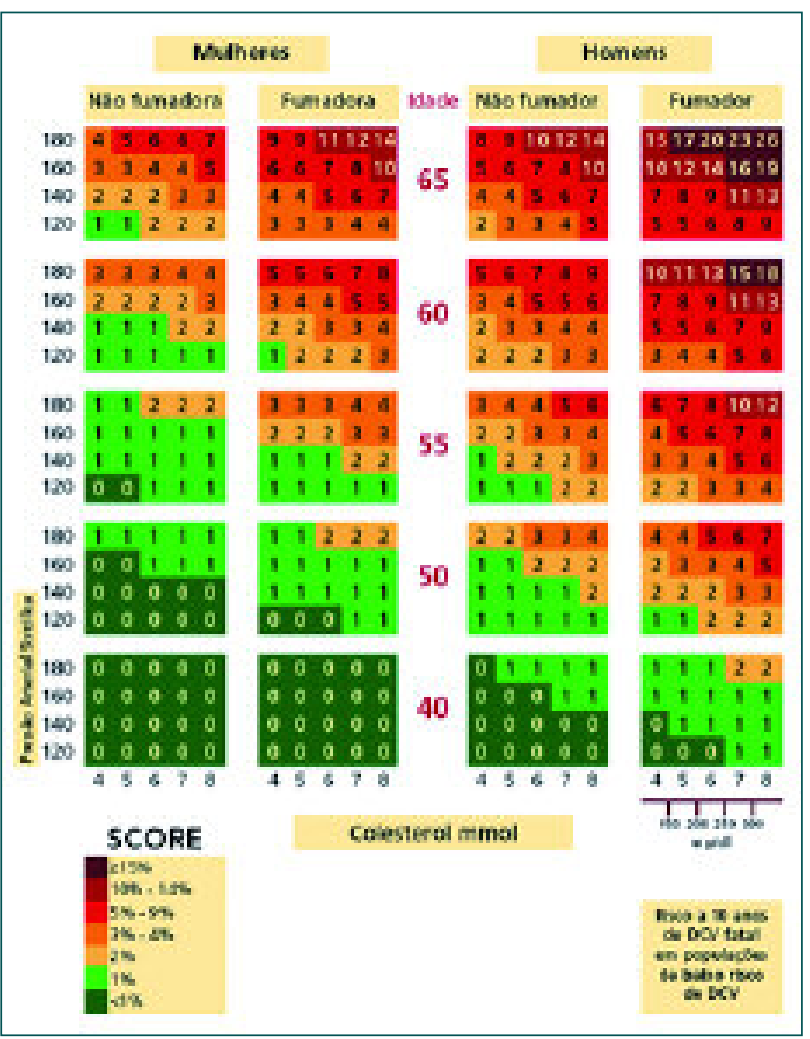

Figura 3. Tabela de cálculo de risco de SCORE.

7. Provavelmente subvaloriza a importância da DM.

8. Não é possível reproduzir os endpoints a partir de outros dados, pelo que é difícil de validar. A definição dos endpoints não fatais usada no estudo de Framingham difere das definições usadas na maioria dos estudos de coorte e em ensaios clínicos (inclui Enfarte Agudo do Miocárdio não fatal, angina de novo e angina instável), o que torna difícil a validação com dados de outros estudos de coorte e a relação com os resultados de ensaios terapêuticos. O risco calculado pela escala de Framingham não pode ser convertido para outras definições. ${ }^{5}$

\section{ESCALA SCORE}

O SCORE foi elaborado por várias sociedades europeias (European Society of Hypertension, European Society of Cardiology, European Society of Atherosclerosis, European Society of General Practice, European Heart Network, European Association for the Study of Diabetes, International Diabetes Foundation, International and Eu- ropean Societies of Behavioural Medicine) ${ }^{6}$

Pretende substituir ou complementar o Framingham nos países europeus. Foi iniciado em 1994 e é baseado em bases de dados muito maiores do que o estudo de Framingham (dados de mais de 250.000 indivíduos, observados durante 10 anos, a partir de doze estudos de coorte que incluem um grande número de países europeus com uma grande área geográfica e diferentes níveis de risco cardiovascular).

Criou folhas de cálculo para as regiões da Europa consideradas de baixo e de alto risco cardiovascular. Este aspecto é importante porque há grandes diferenças na Europa no que respeita às mortes por EAM e AVC. Foram considerados países de baixo risco cardiovascular a Bélgica, França, Itália, Grécia, Luxemburgo, Portugal, Espanha e Suíça. Os restantes países europeus foram classificados como de alto risco.

Esta escala avalia mortalidade por doença cardiovascular total (exclui os eventos não fatais). O valor obtido corresponde ao risco absoluto de doença cardiovascular fatal aos 10 anos (doença coronária fatal + doença não coronária fatal - AVC fatal e doença vascular periférica fatal).

\section{Porque se usaram apenas eventos fatais?}

A morbilidade e a incapacidade que deriva dos eventos não fatais constitui um enorme peso na economia dos sistemas de saúde. Contudo, os eventos não fatais são problemáticos quando usados nos sistemas de cálculo de risco, porque estão muito dependentes das definições e dos métodos usados para o seu diagnóstico.

O estudo Framingham e os sistemas de cálculo do risco que dele derivam incluem, nos endpoints de doença coronária não fatal, para além do EAM não fatal, o início de angina com o esforço e a angina instável. Por isso tem sido difícil, ou mesmo impossível, validar ou reproduzir os endpoints do estudo de Framingham noutros estudos de coorte. A definição usada de doença coronária não fatal não corresponde à usada nos ensaios clínicos.

Assim, de forma a permitir a reprodutibilidade, o SCORE usou como endpoint só a doença cardiovascular fatal.

Outra razão para terem sido considerados apenas os eventos fatais é que o SCORE tem como objectivo, através da criação de folhas de cálculo nacionais e regionais, 
poder ser aplicado em diferentes países da Europa com diferentes taxas de doença cardiovascular e percentagens diferentes de doença coronária e doença não coronária. Muitos países não têm estudos de coorte de doença cardiovascular, mas todos têm dados sobre as causas específicas de mortalidade nacional. Estes dados podem ser usados para estimar o risco da base da população.

O SCORE é constituído por duas tabelas, uma para cada sexo, subdivididas em duas outras - para fumadores e não fumadores. As idades estão organizadas em escalões, assim como os valores do colesterol e da pressão arterial sistólica. As tabelas apresentam maior detalhe nas faixas etárias dos 50 aos 65 anos, período em que as mudanças no risco ocorrem mais rapidamente. Suprimiu-se a faixa etária dos 30 anos, uma vez que estes estão praticamente isentos de risco nos próximos dez anos. Assim, mostrar-lhes o risco poderia dar uma mensagem errada sobre o risco a longo prazo dos indivíduos jovens com factores de risco elevados. ${ }^{5}$

No SCORE existem dois modelos: um baseado no colesterol total; outro na razão colesterol total/colesterol HDL. Pessoas com múltiplos factores de risco tendem a ter níveis de colesterol HDL mais baixos, o que sustenta a preocupação de que ao não considerar o valor do HDL se esteja a subestimar o risco. Contudo, verificou-se que os dois modelos classificam os indivíduos em classes semelhantes e que, por isso, não há grande vantagem em usar o modelo com mais variáveis; está disponível no sistema informático, mas não nas tabelas, porque dificulta o cálculo não se traduzindo em maior acuidade no risco calculado. ${ }^{5} \mathrm{O}$ valor de risco obtido deve ser multiplicado por 4 no caso das mulheres diabéticas e por 2 nos homens diabéticos. Deve ser multiplicado por 1,5 se houver antecedentes pessoais de AVC.

As intervenções terapêuticas não farmacológicas e farmacológicas agressivas são recomendadas para riscos iguais ou superiores a $5 \%$.

Existe também uma tabela de risco relativo.

Os endpoints utilizados são reprodutíveis, o que constitui uma grande vantagem em relação à escala de Framingham. O risco de morte por doença coronária e não coronária (AVC e doença vascular periférica) podem ser avaliados separadamente, através das aplicações informáticas. A doença cardiovascular não coro- nária representa uma grande proporção do total do risco cardiovascular nas regiões da Europa com taxas mais baixas de doença coronária, pelo que o seu cálculo é importante. No entanto, salienta-se que o cálculo do risco cardiovascular total é preferível quando se tomam decisões terapêuticas ou quando se faz educação do doente. $^{5}$

Interactivamente podem introduzir-se dados estatísticos de cada país, o que permite criar folhas de risco nacionais e até regionais, baseadas nos dados da mortalidade de cada região ou país. ${ }^{5}$

\section{Limitações da escala SCORE: ${ }^{1,5}$}

Não prevê a totalidade dos eventos (exclui os não fatais). Folhas de cálculo com limite à inclusão de novas variáveis.

As tabelas não analisam interacções e o impacto da DM e do colesterol HDL necessita de ser melhor estudado.

Baseado numa única medição dos factores de risco e não nos valores habituais.

\section{FRAMINGHAM VERSUS SCORE}

Estas duas escalas usam diferentes parâmetros, pelo que os riscos que calculam são também diferentes (evento coronário fatal e não fatal versus morte cardiovascular) e não podem ser comparados, nem convertidos, entre si. Enquanto a escala de Framingham reflecte a realidade americana, o SCORE reflecte o risco dos países europeus.

Existem limitações comuns às duas escalas: ${ }^{5}$

- Não são considerados parâmetros importantes na avaliação do risco cardiovascular, como sejam: alteração da glicemia em jejum, tolerância diminuída à glicose, obesidade, excesso de peso, medida do perímetro abdominal, sedentarismo, carga tabágica, hipertrofia ventricular esquerda, etnia, hipertrigliceridemia, menopausa prematura, disfunção renal significativa, antecedentes familiares, aterosclerose subclínica, duração da exposição ao factor de risco ou doença.

- O uso de escalões etários pode ser enganador. O risco é inferior ao calculado quando a idade se aproxima do escalão etário anterior, e superior ao calculado quando se aproxima do escalão a seguir.

- Há pouca acuidade no cálculo do risco nos extremos 
etários. O risco está sobrestimado em indivíduos com mais de 70 anos e subestimado em indivíduos com menos de 40 anos.

- Pode haver pouca acuidade no risco calculado para o sexo feminino.

- Pouca acuidade no risco calculado para os diabéticos.

- Não são considerados novos factores de risco, como sejam a PCR de alta sensibilidade, homocisteína, fibrinogénio, apolipoproteína B, lipoproteína a.

\section{RISCO CARDIOVASCULAR NAS MULHERES}

Muitos dos estudos que servem de base aos instrumentos que temos para o cálculo do risco cardiovascular incluíram um número reduzido de mulheres e/ou não foi feita a análise por subgrupos em relação ao sexo. Mulheres com idade superior a 80 anos incluídas nos estudos são em número menor ainda; contudo, nesta faixa etária feminina, a doença cardiovascular é muito comum. ${ }^{1,7}$ Como a esperança de vida das mulheres é superior à dos homens, no futuro teremos maior prevalência da doença nas mulheres, daí a enorme importância de conhecermos a realidade desta no sexo feminino. ${ }^{8}$ São, por isso, necessários estudos só com mulheres ou estudos com mulheres e homens que permitam fazer a análises em separado para os dois sexos.

As mulheres têm a protecção que os estrogénios conferem face à doença cardiovascular durante a idade fértil, mas essa protecção decai após a menopausa, apesar de muitas vezes se manter uma falsa ideia de protecção ligada ao sexo, a qual já desapareceu. ${ }^{8}$ Sabe-se que existem muitas diferenças em relação aos dois sexos no que respeita à doença cardiovascular. Essas diferenças manifestam-se na prevalência dos factores de risco, na forma de aparecimento e de diagnóstico da doença, na evolução, no prognóstico e na resposta à terapêutica. ${ }^{8}$

\section{Diferenças na prevalência dos factores de risco}

A prevalência dos factores de risco nos vários escalões etários é diferente nos homens e nas mulheres. Um número maior de mulheres desenvolve HTA com o aumento da idade, em relação aos homens; apesar da prevalência do tabagismo ser maior nos homens, a diminuição do uso do tabaco é menor nas mulheres; o uso de anticonceptivos orais aumenta ainda mais o risco de fenómenos tromboembólicos no sexo feminino; os ní- veis de colesterol total atingem o máximo entre os $55 \mathrm{e}$ os 65 anos nas mulheres, cerca de uma década mais tarde do que nos homens; a obesidade é mais prevalente nos homens até aos 45 anos, idade após a qual passa a ser mais prevalente nas mulheres; a síndrome metabólica é mais prevalente nas mulheres. ${ }^{1,8}$

\section{Diferenças na idade de aparecimento da doença cardiovascular}

As tabelas parecem indicar que as mulheres têm um risco inferior ao dos homens. Contudo, a única diferença é que o risco delas aparece dez anos mais tarde, devido à protecção dos estrogénios. O risco nas mulheres jovens deve ser por isso extrapolado para os 70 anos em vez de ser para os 60, como acontece no sexo masculino.

A maioria das mulheres com um único, mas severo, factor de risco, terá um risco baixo aos 10 anos. Pelo que, tanto o risco absoluto, como o relativo deve ser calculado, sobretudo no caso das jovens. ${ }^{8}$

\section{Diferenças nas manifestações clínicas}

As manifestações da doença cardiovascular são por vezes diferentes nos dois sexos, podendo haver um subdiagnóstico, por não reconhecimento ou não valorização das queixas da doença nas mulheres. Elas têm maior tendência para ter dor torácica atípica, dor abdominal, dispneia, náuseas, fadiga, isquemia silenciosa. Há maior percentagem de EAM não reconhecido nas mulheres e a prova de esforço apresenta menor acuidade diagnóstica. A doença de vaso único, que é mais frequente no sexo feminino, pode não ser diagnosticada na prova de esforço de rotina. ${ }^{8}$

\section{Diferenças no prognóstico}

A mortalidade das doenças cardiovasculares ajustada à idade diminuiu nos últimos anos, nos países ocidentais. Contudo, analisando esse dados em relação ao sexo, verifica-se que essa diminuição é menor nas mulheres, as quais têm maior mortalidade nos sindromas coronários agudos e pior prognóstico nos AVC do que os homens. ${ }^{1,8}$

\section{Diferenças na resposta à terapêutica}

As diferenças na resposta à terapêutica explicam-se, entre outros aspectos, pelos diferentes níveis hormonais, menor peso, maior percentagem de gordura corporal, diferenças na actividade enzimática e na taxa de filtra- 
ção glomerular das mulheres em relação aos homens. O exemplo das diferenças na resposta às terapêuticas cardiovasculares, em relação ao sexo, é representado pela aspirina na prevenção primária do EAM e do AVC: nas mulheres a aspirina diminui o risco de AVC mas não o do EAM; nos homens, ao contrário, a aspirina reduz o risco de EAM mas não o de AVC, condicionando até um aumento não significativo do risco deste último. Não se sabe exactamente o porquê destas diferenças, mas elas vêm alertar para a importância de ensaios clínicos que tenham um número representativo de mulheres. ${ }^{8}$

\section{TERAPÊUTICA HORMONAL DE SUBSTITUIÇÃO (THS) E RISCO CARDIOVASCULAR}

Os estudos HERS (Heart and Estrogen Replacement Study, NHS (Nurses Health Study) e WHI (Women's Health Iniciative) vieram confirmar que a terapêutica hormonal não está recomendada para protecção cardiovascular. Ela serve apenas para alivio dos sintomas da menopausa. ${ }^{7,8}$ Os diferentes regimes de terapêutica hormonal de substituição têm efeitos muito diferentes a nível cardiovascular, uma vez que diferem entre si nos seus efeitos metabólicos. ${ }^{8}$

Do resultado destes estudos, permanece a dúvida se o aumento do risco cardiovascular se deve ao efeito da combinação de estrogénio/progestativo, ao tipo de progestativo ou às doses usadas. Estas questões não estão respondidas, pelo que ainda é necessária mais investigação em relação à dose ideal, tipo de terapêutica, via de administração e duração da terapêutica hormonal para um benefício máximo, com um risco mínimo. ${ }^{8}$

Estão a decorrer mais estudos sobre o efeito da THS em prevenção primária de doença cardiovascular. O recentemente publicado estudo WISDOM veio também confirmar o aumento do risco cardiovascular causado pela THS, iniciada muitos anos após a menopausa, em mulheres sem doença cardiovascular. ${ }^{9}$ Contudo, para muitas mulheres, a terapêutica de curto tempo para alívio dos sintomas menopáusicos justifica o pequeno aumento absoluto do risco de doença coronária e de neoplasia da mama. O risco destas complicações aumenta com a duração da THS, pelo que esta deve ser prescrita durante o menor tempo possível. ${ }^{8}$

Os moduladores dos receptores de estrogénios (raloxifeno e tamoxifeno) também não devem ser usados na prevenção da doença coronária. Não só são inefica- zes a proteger o coração, como podem aumentar o risco de AVC. ${ }^{10}$

\section{RISCO CARDIOVASCULAR NOS IDOSOS}

Há poucos estudos na população idosa, em particular nas mulheres. No estudo Framingham o número de idosos foi pequeno, pelo que neles pode faltar acuidade no cálculo do risco. ${ }^{1}$

O risco das faixas etárias mais elevadas está sobrevalorizado. Os idosos têm um risco absoluto elevado, dado o peso do factor idade. Isto pode conduzir ao sobreuso de fármacos. Por isso, as European Guidelines on CVD Prevention recomendam que nos idosos se considere o tratamento farmacológico só quando o SCORE é igual ou superior a $10 \%{ }^{1}$

O estudo PROSPER (Prospective Study of Pravastatin in the Elderly at Risk), que compara a terapêutica com estatinas nos idosos e nos jovens, realça que o uso de estatinas nos idosos é custo-eficaz, sobretudo por causa do risco mais elevado neste grupo etário. ${ }^{11}$ Os idosos atingem maiores reduções do colesterol LDL que os jovens, com a mesma dose de estatina. No entanto, também estão mais predispostos aos efeitos indesejáveis. Há evidência de que os doentes com doença cardíaca e com mais de 65 anos, incluindo os com mais de 80 anos, têm reduções da mortalidade cardiovascular com a terapêutica com estatinas. Mas falta evidência disto em relação ao uso das estatinas na prevenção primária nos doentes com mais de 80 anos.

\section{RISCO CARDIOVASCULAR NOS DIABÉTICOS}

A Diabetes aumenta consideravelmente o risco cardiovascular. Na Europa, a causa mais comum de morte no diabético é a doença coronária. ${ }^{12}$ Este aumento do risco depende de factores como o tipo e duração da DM, o controlo glicémico e a presença de retinopatia, micro-albuminúria ou proteinúria.

A avaliação do risco de DM deve fazer parte da avaliação do risco cardiovascular global. A relação entre a hiperglicemia e a doença cardiovascular deve ser vista como um contínuo. O risco cardiovascular aumenta com o aumento da Hemoglobina Alc. ${ }^{12}$

As perturbações do metabolismo glucídico conferem um risco particularmente elevado em relação à mortalidade e morbilidade cardiovascular nas mulheres. ${ }^{13,14}$

A glicemia pós-prandial fornece melhor informação 
do risco cardiovascular futuro que a glicemia em jejum. A prevalência da Tolerância Diminuída à Glicose Oral aumenta linearmente com a idade, enquanto que a Alteração da Glicemia em Jejum não. ${ }^{12}$

Nos indivíduos com glicemia em jejum normal, uma glicemia pós-prandial aumentada prediz um aumento do risco cardiovascular. ${ }^{12}$ Estão a surgir estudos que demonstram que o controlo da hiperglicemia pós-prandial diminui o risco cardiovascular. O Estudo DECODE - Diabetes Epidemiology Collaborative Analysis of Diagnostic Criteria in Europe - demonstrou que tanto a glicemia em jejum como a glicemia pós-prandial são factores de risco independentes para a mortalidade e morbilidade cardiovascular e por todas as causas, mesmo nos indivíduos sem DM diagnosticada. ${ }^{12}$

Nos indivíduos com doença cardiovascular que têm alteração do metabolismo glucídico, muitas vezes é a glicemia pós-prandial que está alterada e não a glicemia em jejum. Assim, nestes doentes deve avaliar-se tanto a glicemia em jejum como a prova de tolerância à glicose oral. ${ }^{12}$

Vários estudos na Europa demonstraram que a presença da síndroma metabólica (SM) aumenta a mortalidade por todas as causas e a mortalidade por doença cardiovascular, mas alguns estudos dos EUA mostraram evidências inconsistentes. ${ }^{12} \mathrm{ASM}$ identifica pessoas com risco mais elevado de doença cardiovascular que a população em geral, apesar de não ser melhor, ou sequer igual, preditor de risco cardiovascular que os scores baseados nos factores de risco major. Há também evidência de que a DM é, ela própria, um factor de risco cardiovascular independente dos factores de risco que a constituem. Contudo, estas são ainda áreas em estudo. ${ }^{12}$

O estudo de Framingham, para além de incluir um número muito reduzido de diabéticos (4\% do total), usou a sua própria definição de DM, pelo que o considerar o factor de risco diabetes como uma variável dicotómica tem sido questionado, pondo em causa a sua validação. ${ }^{5}$ Os estudos de coorte que serviram de base ao SCORE não recolheram de forma uniforme os dados em relação à DM, pelo que não se podiam aplicar os critérios diagnósticos da Associação Americana de Diabetes, nem os da Organização Mundial de Saúde. Por isso, não se incluiu variável dicotómica, nem se usaram folhas de cálculo distintas para os diabéticos. As folhas de risco do SCORE podem, contudo, ser utilizadas porque, a relação entre os factores de risco convencionais e a doença cardiovascular é paralela nos diabéticos e nos não diabéticos, apesar do risco nos diabéticos ser muito maior. O SCORE multiplica o risco obtido por 2 nos homens com DM, e por 4 nas mulheres com Diabetes. ${ }^{5}$

O estudo DECODE (Diabetes Epidemiology Collaborative Analysis of Diagnostic Criteria in Europe) criou uma equação para cálculo do risco de doença cardiovascular fatal que inclui estado de tolerância à glicose e glicemia em jejum. No entanto, também é pouco fiável, uma vez que usa a glicemia em jejum como categoria, não considerando o efeito dos diferentes níveis de glicemia. ${ }^{14,15}$

A revista Diabetes Care publicou, em 2007, um estudo que compara Framingham, SCORE e equações de risco do estudo DECODE. Verificou-se que nenhuma das três dá uma estimativa confiável do risco cardiovascular nos diabéticos tipo 2. Futuros estudos têm que incluir variáveis relacionadas com a DM, para além dos factores de risco convencionais. ${ }^{15}$

O UKPDS já desenvolveu modelos matemáticos que estimam o risco absoluto de doença coronária e AVC em doentes com DM tipo 2 recém diagnosticada. O UKPDS 56 estima o risco de doença coronária e usa como parâmetros: glicemia, hemoglobina A1C como variável contínua, pressão arterial sistólica, idade em que é feito o diagnóstico, sexo, grupo étnico, tabagismo, tempo que decorreu desde o diagnóstico, colesterol total/HDL. ${ }^{16} \mathrm{O}$ UKPDS 60 estima o risco de AVC e inclui duração da diabetes, idade, sexo, tabagismo, pressão arterial sistólica, colesterol total/HDL, fibrilhação auricular. Porém, e apesar de serem um avanço, estes modelos matemáticos ainda não estão disponíveis sob a forma de instrumentos de trabalho aplicáveis na consulta. ${ }^{17}$

Até novas directrizes, devemos considerar os diabéticos como doentes de alto risco (se ainda não o são, têm grande probabilidade de o vir a ser, no futuro).

\section{CONCLUSÕES}

O cálculo do risco cardiovascular global é essencial na prática clínica actual e é dinâmico. Deve ser realizado de forma regular e revisto sempre que houver alteração nos factores de risco existentes.

O valor obtido deve ser alvo de ponderação pelo médico, com vista a considerar aspectos que não são tidos em conta nas escalas, fazendo uso do senso clínico e adequando as decisões ao indivíduo em causa. 
As escalas de risco existentes, apesar das muitas limitações que têm, são valiosos instrumentos de trabalho, que permitem que as decisões médicas não sejam arbitrárias, mas sim assentes em estudos e baseadas na evidência.

\section{REFERÊNCIAS BIBLIOGRÁFICAS}

1. Graham I, Atar D, Borch-Johnsen K, Boysen G, Burell G, Cifkova R, et al. European Guidelines on Cardiovascular Disease Prevention in Clinical Practice: executive summary. Fourth Joint Task Force of the European Society of Cardiology and Other Societies on Cardiovascular Disease Prevention in Clinical Practice. Eur Heart J 2007 Oct; 28 (19): 2375-414.

2. Grundy SM, Cleeman JI, Merz CN, Brewer HB Jr, Clark LT, Hunninghake DB, et al. Implications of recent clinical trials for the National Cholesterol Education Program Adult Treatment Panel III guidelines. Circulation 2004 Jul 13;110 (2): 227-39.

3. Direcção-Geral da Saúde. Direcção de Serviços de Prestação de Cuidados de Saúde. Divisão de Qualidade. Risco Global Cardiovascular. Lisboa: Direcção-Geral da Saúde; 2007. Disponível em: URL: http://www. dgs.pt/default.aspx?cr=11179 [acedido em 03/06/2008].

4. Wilson PW, D'Agostino RB, Levy D, Belanger AM, Silbershatz H, Kannel WB. Prediction of coronary heart disease using risk factor categories. Circulation 1998 May 12; 97 (18): 1837-47.

5. Conroy RM, Pyörälä K, Fitzgerald AP, Sans S, Menotti A, De Backer G, et al. Estimation of ten-year risk of fatal cardiovascular disease in Europe: the SCORE project. Eur Heart J 2003 Jun; 24 (11): 987-1003.

6. Brookes L. European Guidelines on Cardiovascular Disease Prevention [Internet] 2003. Disponível em: URL: http://www.medscape.com/viewarticle/462539 [acedido em 03/06/2008]

7. Mosca L,Appel LJ, Benjamin EJ, Berra K, Chandra-Strobos N, Fabunmi RP, et al. AHA Guidelines. Evidence-based guidelines for cardiovascular disease prevention in women. Circulation 2004 Feb 10; 109 (5): 672-93.

8. Stramba-Badiale M, Fox KM, Priori SG, Collins P, Daly C, Graham I, et al. Cardiovascular diseases in women: a statement from the policy conference of the Eu- ropean Society of Cardiology. Eur Heart J 2006 Apr; 27 (8): 994-1005.

9. Vickers MR, MacLennan AH, Lawton B, Ford D, Martin J, Meredith SK, et al. Main morbidities recorded in the women's international study of long duration oestrogen after menopause (WISDOM): a randomised controlled trial of hormone replacement therapy in postmenopausal women. BMJ 2007 Aug 4; 335 (7613): 239-50.

10. Mosca L, Banka CL, Benjamin EJ, Berra K, Bushnell C, Dolor RJ, et al. Evidence-based guidelines for cardiovascular disease prevention in women: 2007 update. Circulation 2007 Mar 20; 115 (11): 1481-501.

11. Shepherd J, Blauw G, Murphy M. The PROSPER Trial. Lancet 2003 Feb 1; 361 (9355): 427-8.

12. Rydén L, Standl E, Bartnik M, Van den Berghe G, Betteridge J, Boer M, et al. Guidelines on diabetes, pre-diabetes, and cardiovascular diseases: executive summary. The Task Force on Diabetes and Cardiovascular Diseases of the European Society of Cardiology (ESC) and of the European Association for the Study of Diabetes (EASD). Eur Heart J 2007 Jan; 28 (1): 88-136.

13. Cardiovascular Risk: assessment and management. Disponível em: URL: http://cks.library.nhs.uk/cardiovascular_risk/ [acedido em 03/06/2008].

14. Martins AS, Coelho AS, Rodrigues E, Polónia J, Medina JL, Silva JM, et al. Risco cardiovascular global. Lisboa: Bayer, s/d.

15. Coleman RL, Stevens RJ, Retnakaran R, Holman RR. Framingham, SCORE, and DECODE risk equations do not provide reliable cardiovascular risk estimates in type 2 diabetes. Diabetes Care 2007 May; 30(5):1292-3.

16. Stevens RJ, Kothari V, Adler Al, Stratton IM. The UKPDS risk engine: a model for the risk of coronary heart disease in Type II diabetes (UKPDS 56). Clin Sci (Lond) 2001 Dec; 101 (6): 671-9.

17. Kothari V, Stevens RJ, Adler Al, Stratton IM, Manley SE, Neil AH, et al. UKPDS 60: risk of stroke in type 2 diabetes estimated by the UK Prospective Diabetes Study Risk Engine. Stroke 2002 Jul; 33 (7): 1776-81.

18. RCMAR Measurement Tools. Coronary Heart Disease Risk Assessment. Disponível em: URL: http://www.ncbi.nlm.nih.gov/books/bv.fcgi?rid= citmed.part.1327 [acedido em 03/06/2008]

19. Mancia G, De Backer G, DominiczakA, Cifkova R, Fagard R, Germano G, et al. 2007 Guidelines for the management of arterial hypertension: The Task Force for the Management of Arterial Hypertension of the European Society of Hypertension (ESH) and of the European Society of Cardiology (ESC). Eur Heart J 2007 Jun; 28 (12): $1462-536$.

\section{ABSTRACT}

Introduction: The risk of suffering from atherosclerotic illness and its cardiovascular complications, which is named global cardiovascular risk, may be evaluated using several risk scale-based methods. These are taken into account along with several interactive risk factors and display some advantages and limitations which are worthy of consideration. The objective of cardiovascular risk evaluation is to identify groups of individuals which must be advised and treated with the goal of preventing cardiovascular illness, as well as to establish the level of therapeutic aggressiveness to enforce. We have included considerations on risk groups which have particular characteristics, namely women, elderly people and diabetic patients.

Methodology: A survey was conducted through on-line databases, and key documental sources in the areas of Cardiology, General and Family Medicine and Internal Medicine were researched, not excluding the recommendations from various international organizations concerning the evaluation of cardiovascular risk.

Conclusions: The rising significance of mortality and morbidity related with cardiovascular atherosclerotic illness turns the evaluation of global cardiovascular risk into an essential process of current clinical practice. The calculation of this risk must be regular and dynamic, and revision is compulsory whenever the pending risk factors are altered. The obtained value must be pondered by the doctor; aspects not regarded by the risk scales must be considered, resorting to clinical good judgment and adapting the decisions to the individual concerned. The current risk scales are valuable instruments, despite their limitations, thus contributing to medical decisions supported by studies and evidence based.

Keywords: Global Cardiovascular Risk; SCORE; Framingham; Cardiovascular Risk in Women; Cardiovascular Risk in Diabetic Patients; Cardiovascular Risk in Elderly People. 